The rotation may be in a clockwise or anti-clockwise direction.

Altogether five species of monoflagellates have been mvestigated, and three of them filmed at considerable length. The action of the flagellum in all five species appears to be identical in outline, though quite different in detail.

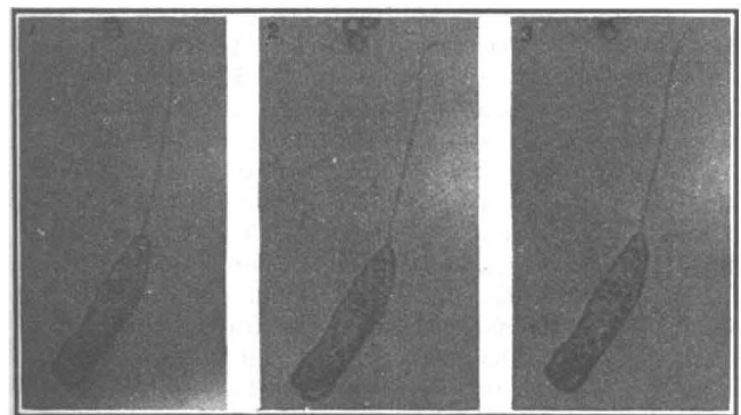

FIG. 3. Peranema trichophorum. Three consecutive exposures. Time of exposure, $1 / 8,000$ sec. Interval, $1 / 30$ sec. approx. The waves are confined to the tip of the flagellum. In this case, the organism is swimming forwards with its maximum speed. The extreme tip of the flagellum is not seen since it lies beyond the penetration of the objective.

To sum up: (1) The flagellum generates its own energy, at any rate in part, if not completely. (2) The waves of distortion pass from base to tip of the flagellum and not in the reverse direction. (3) The actual position taken up by the flagellum at any instant will determine whether these waves of distortion constitute a forward or backward com. ponent. (4) They may also cause the organism to rotate in a clockwise or anti-clockwise direction. (5) They constitute an important escape movement by causing the organism to reverse its direction

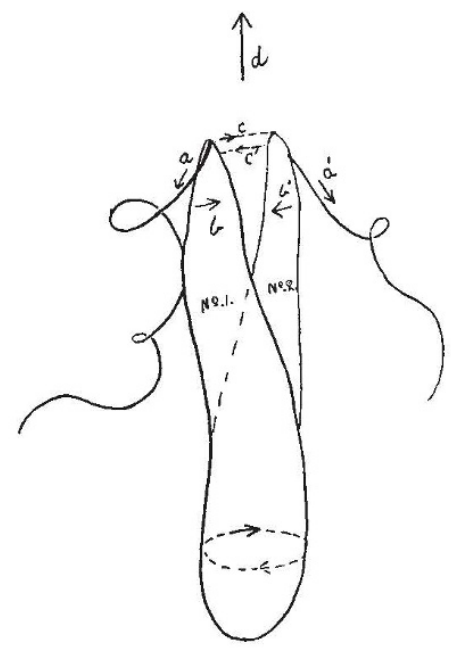

FiG. 4. Diagrammatic representation of Euglena viridis.

No. 1. A wave is passing along the flagellum in a direction $a$. This causes the organism to rotate in a clockwise direction $b$. It also causes lateral disp. It also causes lateral displacethe animal $c$. No. 2. The flagellum is organism. wave or cither the same ave or another passes along the flagellum. Lateral displacement is in the direction $c^{\prime}$. The organism is propelled in the direction $d$, and rotates in a clockwise direction. The anterior end moves in a ircular path at right angles to the mean direction.

rapidly without necessitating a reversal in either the direction of propagation of the waves themselves or the direction of rotation of the organism.

'J. Gray, "A Text-Book of Experimental Cytology". Cambridge University Press. 1931.

"A. G. Lowndes, "The Twin Polygraph and Strobograph", Nature, 135, 1006 (June 15, 1935).

\title{
Specific Heats of Metals and Alloys at High Temperatures*
}

\begin{abstract}
A FTER some ten years of experience in the $A$ investigation of various physical properties of metals at high temperatures, Prof. F. M. Jaeger and his collaborators at Groningen commenced, about fifteen years ago, the much more difficult investigation of the changes which the specific heats of metallic substances undergo at temperatures up to $1,600^{\circ} \mathrm{C}$. At that time there were considerable differences in the data published by various experimenters regarding the specific heats of metals and their temperature coefficients at those high temperatures-differences which could not be accounted for entirely by variations in experimental technique, but which seemed to be due, in part at least, to unknown peculiarities of the metals themselves. As a result of the work of Prof. Jaeger, many of these discrepancies have been removed and much valuable information has been obtained concerning the nature of thermal and molecular transformations in the metallic state.

The direct specific heat measurements were carried out by means of a high-precision metal-block calorimeter in which temperature differences of a few millionths of $1^{\circ} \mathrm{C}$. could be measured with certainty. The results obtained by the calorimetric technique were checked and confirmed by independent methods, for example, by measurements of the variation with temperature of the electrical resistance and of the

- Substance of a lecture by Prof. F. M. Jaeger, University of Groningen, delivered at University College, London, on May 11.
\end{abstract}

thermo-electric behaviour of the metals. In studying the variation of any such property as a function of temperature, use was also made of two optically coupled galvanometers combined with a photographic recording device in order to obtain continuous curves, which immediately reveal any sudden discontinuity of the variable property at a particular temperature.

As regards the discrepancies in the results of previous workers, Prof. Jaeger's investigations show that these can be largely attributed to the measurements having been made with samples of metal which had undergone different preliminary thermal or mechanical treatment and which, therefore, were physically non-homogeneous due to the presence of inner stresses. Metals which have been suitably tempered by prolonged heating in vacuo at a constant high temperature as near to the melting point as possible, followed by slow cooling in vacuo, give values of the specific heat which, even at lower temperatures, are quite reproducible. This is especially striking in the case of silver, for which Prof. Jaeger has obtained a $C_{p}$-temperature curve which is concave to the temperature axis and not convex, as indicated by the best previously recorded data.

In many cases, the behaviour of metals at high temperatures is complicated by the occurrence of allotropy, and the calorimetric method is particularly suited to the detection of such changes. The trans- 
formation to a new allotropic form usually commences at a much lower temperature than the thermodynamically defined transition point, and in this 'transition interval' the specific heat of the metal increases very rapidly with the temperature, often reaching extremely high values.

In passing through the transition point, either by heating or by cooling, the allotropic changes are frequently accompanied by certain retardation phenomena. For example, beryllium, which has been given a preliminary heating at temperatures of $450^{\circ} \mathrm{C}$. or more and is then allowed to cool from $400^{\circ} \mathrm{C}$., gives off the same amount of heat, but over a longer period, than a sample of the metal which has not been heated above $400^{\circ} \mathrm{C}$. The change in the internal state of the metal brought about by the preliminary heating may persist for several months, but there is a continuous return to the original state. Similar changes have been observed with rhodium, zirconium, chromium, titanium, and other metals, and the phenomenon appears to be a very general one. It is interesting to note that in.the case of beryllium, the retardation of heat evolution com. pletely disappears if the sample of metal is previously finely powdered.

Another factor of importance is the remarkable influence which may be exerted by almost imper. ceptible traces of gases-chiefly of oxygen, but occasionally also of nitrogen and of hydrogen-on the phase changes which occur in metals. In the $\alpha$-titanium $\rightleftarrows \beta$-titanium transition, for example, there is at the transition temperature of $882^{\circ}$ an additional hysteresis effect, which disappears with a sample of metal prepared in vacuo, but returns on the addition of traces of oxygen and nitrogen.

Prof. Jaeger's experimental study of the specific heats of metals at different temperatures has provided more accurate data which can be applied to testing the validity at high temperatures of two important theoretical deductions. According to the quantum-theoretical treatment by Einstein and by Debye of the variation of the specific heat of solid substances with temperature, the atomic heat at constant volume of solid mono-atomic elements should, even at the highest temperatures, never exceed a value of $3 R=5 \cdot 955$ cal. The experimental results make it improbable, however, that any such upper limit exists, since this value is already ex. ceeded in most cases at relatively low temperatures. The second application concerns the validity of the Neumann-Kopp law, which states that the molecular heat of a compound is an additive property of the atomic heats. Prof. Jaeger's measurements show that, in the case of alloys, the law is obeyed only when true solid solutions are formed between the component metals. With intermetallic compounds, the deviations from additivity, which are sometimes very considerable, may be either positive or negative, and in all cases rapidly increase with the temperature of observation.
O. J. WALKER.

\section{Insect Pests of Crops in England*}

$\mathrm{T}$ 'HE Ministry of Agriculture and Fisheries' report for 1932-34 on insects affecting crops has been drawn up by Mr. J. C. F. Fryer, in collaboration with his colleagues at the Plant Pathological Laboratory, Harpenden. In dealing with advances in the applica. tion of insecticidal measures, the subject of pyrethrum is again considered in this report. Methods for the evaluation of the toxic principles of pyrethrum, elaborated by Dr. F. Tattersfield at the Rothamsted Station, have proved valuable, and it has been further shown that the incorporation of certain antioxidants greatly retards the deterioration of pyrethrum dust when exposed to the air. The plant has been shown to grow, and to yield satisfactorily, under English conditions; but the available methods of harvesting entail too heavy labour costs to warrant cultivation as a paying proposition. This aspect, therefore, remains in abeyance, at any rate for the time being, but in view of the valuable properties of pyrethrum, and the safety attending its application as a spray fluid, we may hope that cultural difficulties will eventually be overcome.

The hot-water treatment of daffodil bulbs as a measure against the Merodon fly and nematode pests is now an established procedure, and the same method has now become applicable in respect to the Tar. sonemus mite of the strawberry. The treatment has been further developed with reference to strawberries, chrysanthemums and violets.

Among introduced pests, the Colorado potato

* Ministry of Agriculture and Fisheries. Bulletin No. 99 : Report on Insect Pests of Crops in England and Wales, 1932-1934. Pp. vi +50 . (London: H.M. Stationery Office, 1936.) 18, net. beetle is of great importance : the recent eradication of a slight infestation in the Tilbury-Gravesend area is a matter for congratulation to all concerned. But for the prompt measures put in force by the Ministry, this insect might have gained a foothold, and led to a veritable campaign for its eradication. The extent of its adopted area in France, and its continued spread in that country, will serve as a justification for the close scrutiny that is being maintained at likely English ports of ingress.

Among other foreign pests that have found entry into Great Britain, reports on the eradication of the chrysanthemum midge and fluted scale are satisfactory, and measures to preclude the introduction of the apple maggot and cherry fruit fly have given the effective results anticipated. During the period under review, many indigenous crop pests are men. tioned. There were no very outstanding attacks on cereals but with regard to potatoes, the Heterodera eelworm was a serious menace and appeared to be spreading. Larvæ of chafer beetles were specially in evidence as pests of grassland, while in connexion with sugar beet cultivation, the discovery of the Heterodera potato eelworm in that crop has complicated the situation. Proper rotation and clean cultivation may avert the severe losses which this creature has brought about on the continent of Europe. Brassica crops came in for heavy infestation by the aphis Brevicoryne, flea beetles and the cabbage white fly - dry weather conditions seemingly having favoured such attacks.

Fruit pests are always numerous : the apple sawfly attracted most attention, but the application of 